\title{
Das Bronchialkarzinom - Herausforderung für den Kliniker
}

D as Bronchialkarzinom fordert unter den malignen Tumoren die meisten Todesopfer. Für das Jahr 2000 wurden weltweit zwischen 1,3 und 2 Millionen Todesfälle geschätzt. In den 90er Jahren starben jährlich in den USA rund 170000 und in Deutschland etwa 37000 Menschen an diesem Tumor.

Im Verlauf der letzten Dekade wurden Konzepte in der Behandlung von Patienten mit nichtkleinzelligen Bronchialkarzinomen entwickelt, welche frühere Auffassungen revidierten und ein zunehmendes Interesse an dieser Tumorentität weckten. Deutlich wird dies an der Vielzahl von Publikationen und wissenschaftlichen Beiträgen auf internationalen Kongressen. Unzweifelhaft ist, dass die Therapie der Wahl beim nichtkleinzelligen Bronchialkarzinom, wenn es zum Zeitpunkt der Diagnose noch resektabel erscheint, in der kurativen Operation besteht. Doch auch wenn eine radikale Resektion möglich ist, lassen sich mit den modernen chirurgischen Verfahren nur kumulative Fünf-Jahres-Überlebensraten von $50-60 \%$ im Stadium pTI erreichen. Noch schlechter ist die Situation in höheren Stadien: Im Stadium pTII betragen die kumulativen Fünf-Jahres-Überlebensraten nur noch etwa $40 \%$, im Stadium pTIIla noch $25 \%$ und im Stadium pTIIIb liegen sie sogar unter $20 \%$. Demzufolge kann die chirurgische Therapie nur bei einer Minderheit der Patienten kurativ sein.

Aus Analysen des Rezidiv- und Metastasierungsmusters nach der Operation müssen wir schließen, dass Bronchialkarzinome bei der größten Zahl der Patienten bereits von vornherein disseminiert, zum Zeitpunkt der Diagnose lediglich nicht als fernmetastasiert erkennbar sind. In dieser Situation klingt es logisch, bereits primär eine systemische Chemotherapie einzuleiten, und aufgrund der Ergebnisse klinischer Studien der letzten Jahre scheint sich dieses Konzept auch allseits durchzusetzen. Sowohl mit der präoperativen als auch mit der postoperativen adjuvanten Chemotherapie können die FünfJahres-Überlebensraten um ein Ausmaß verbessert werden, das dem anderer solider Tumoren entspricht.

Hinzu kommen auch eindeutige Verbesserungen in der Behandlung von Patienten mit fortgeschrittenen disseminierten nichtkleinzelligen Bronchialkarzinomen im Stadium IV, für die nur noch palliative Behandlungsmöglichkeiten der Chemotherapie und/oder Radiotherapie bestehen. Bis vor wenigen Jahren galt noch allgemein die Meinung, beschwerdefreie Patienten dieses Stadiums nach Möglichkeit nicht zu behandeln und nur zu betreuen. Heute jedoch besteht eine immer höhere Bereitschaft, bei diesen Patienten - wenn ihre biologischen Voraussetzungen dies erfüllen - sofort mit dem Beweis der Fernmetastasierung eine Chemotherapie einzuleiten. Die Leitlinien wissenschaftlicher Fachgesellschaften haben dieses Vorgehen inzwischen dokumentiert. Möglich wurde die Änderung unserer Einstellung durch die Entwicklung neuer, besser verträglicher Zytostatika und von Medikamenten mit anderen Wirkungsmechanismen wie monoklonale Antikörper und Inhibitoren des Epidermal-growth-factor-Rezeptors (EGFR).

Anders ist die Situation beim kleinzelligen Bronchialkarzinom, denn in dieser Indikation besteht derzeit eine gewisse Stagnation in der Entwicklung der Therapieverfahren - allerdings auf einem wesentlich höheren Niveau als noch vor 15-20 Jahren.

Mit dem aktuellen Schwerpunktheft des klinikarzt zum Thema Bronchialkarzinom haben wir uns zum Ziel gesetzt, der in der Ärzteschaft noch weit gehend vorhandenen Unkenntnis über die modernen Entwicklungen der multimodalen potenziell kurativen Therapieansätze und Möglichkeiten einer palliativen Therapie zu begegnen. Denn nicht nur für den klinischen Forscher wurde das Bronchialkarzinom im Verlauf der letzten Dekade zu einem interessanten Tumor, es fordert vielmehr auch den in der Praxis und der Klinik tätigen Arzt deutlich mehr als früher.

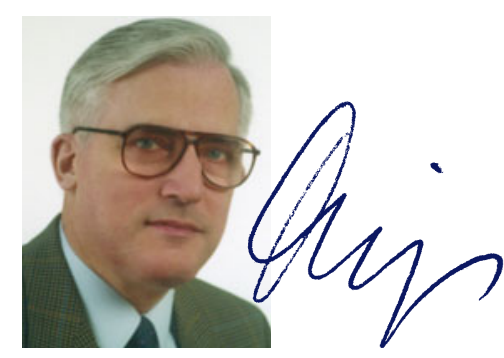

Prof. Dr. Peter Drings, Heidelberg 